\title{
Signs of life in Q2
}

\section{Walter Yang}

Last quarter, the major stock indexes were up, and investors got back into the game of funding publicly traded biotechs. The industry raised $\$ 4.6$ billion, excluding partnership monies, compared with only $\$ 2$ billion in 4Q08 at the height of the credit crisis. Through follow-ons and

\section{Stock market performance}

The tech-heavy NASDAQ Composite led the rally last quarter, posting a $20 \%$ gain. The BioCentury 100 and the NASDAQ Biotechnology indexes were up $12 \%$ and $10 \%$, respectively.

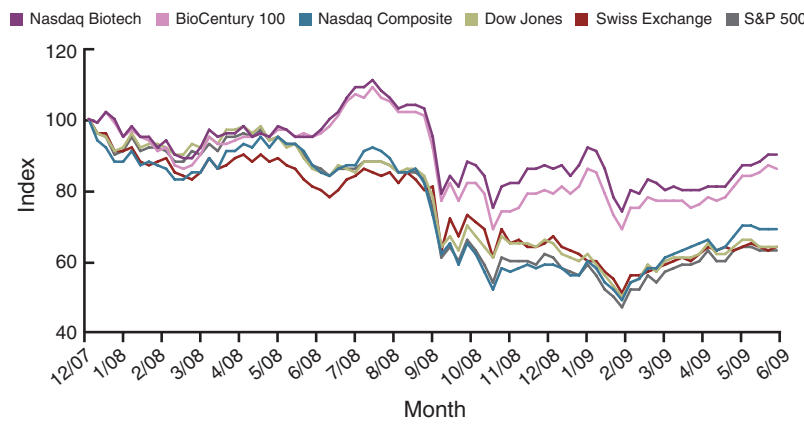

\section{Global biotech industry financing}

Compared with $\$ 264$ million in 4Q08, public biotechs raised $\$ 1.4$ billion through equity securities, including follow-ons and PIPEs.

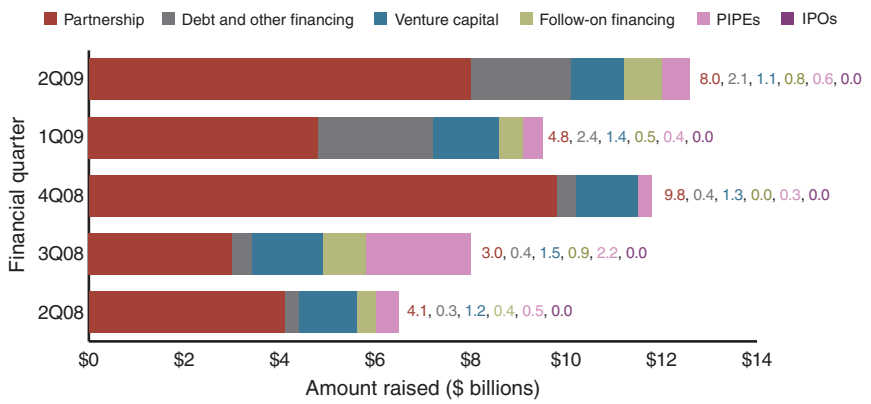

IPO, initial public offering; PIPEs, private investments in public equity
PIPEs (private investments in public equity), biotechs raised $\$ 1.4$ billion in 2Q09 versus \$264 million in 4Q08. Debt financings were also up considerably, including Cephalon's $\$ 500$ million in convertible notes. Private biotech funding also remained robust.

\section{Global biotech initial public offerings}

Only four companies have completed IPOs in the past five quarters.

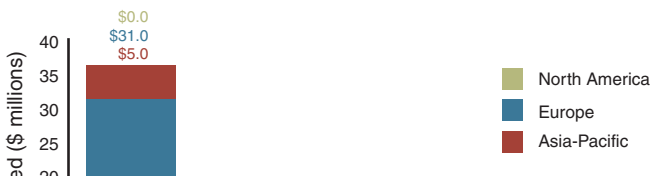

Asrope

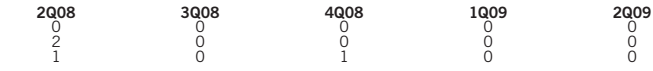

Table indicates number of IPOs. Source: BCIQ: BioCentury Online Intelligence

\section{Global biotechnology venture capital investment}

Private biotechs raised $\$ 1.1$ billion in 2Q09, slightly off (9\%) from 2Q08.

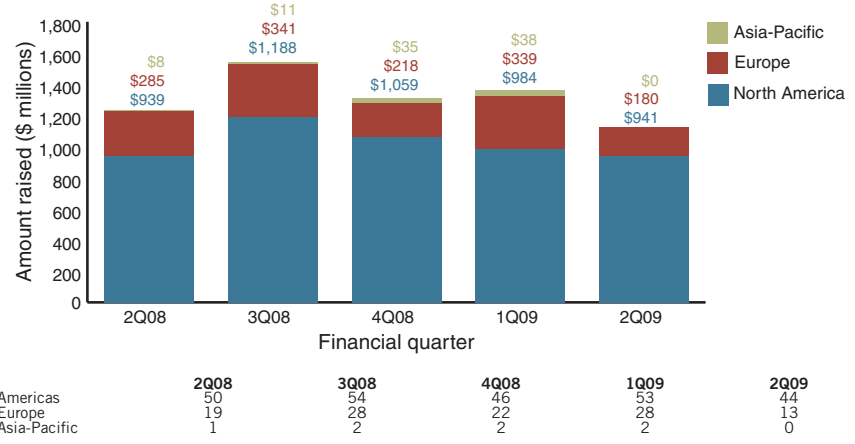

Table indicates number of VC investments and includes rounds where the amount raised was not disclosed. Source: BCIQ: BioCentury Online Intelligence

\begin{tabular}{|c|c|c|c|c|c|c|c|c|c|}
\hline \multirow[t]{9}{*}{$\begin{array}{l}\text { Venture } \\
\text { capital }\end{array}$} & \multirow{3}{*}{$\begin{array}{l}\text { Company (lead investors) } \\
\text { Clovis Oncology }{ }^{1} \text { (Domain, New Enterprise Associates, Versant, Aberdare, } \\
\text { Abingworth, Frazier Healthcare, ProQuest, company management) } \\
\text { Hyperion (Bay City Capital, Panorama Capital) }\end{array}$} & $\begin{array}{l}\text { Amount raised } \\
\text { (\$ millions) }\end{array}$ & $\begin{array}{c}\text { Round } \\
\text { number }\end{array}$ & Date closed & $\begin{array}{l}\text { Mergers and } \\
\text { acquisitions }\end{array}$ & Target & \multirow{2}{*}{$\begin{array}{l}\text { Acquirer } \\
\text { Johnson \& Johnson }\end{array}$} & \multirow{2}{*}{$\begin{array}{c}\begin{array}{c}\text { Value } \\
\text { (\$ million) }\end{array} \\
1,000.0\end{array}$} & \multirow{2}{*}{$\begin{array}{l}\text { Date announced } \\
\text { 21-May }\end{array}$} \\
\hline & & 145.0 & NA & 21-May & & Cougar Biotechnology & & & \\
\hline & & 60.0 & 3 & 30-Jun & & BiPar & Sanofi Aventis & 500.0 & 15-Apr \\
\hline & Novlmmune (BZ Bank) & 56.4 & NA & 12-May & & Monogram Biosciences & $\begin{array}{l}\text { Laboratory Corp. } \\
\text { of America }\end{array}$ & 106.7 & 23-Jul \\
\hline & $\begin{array}{l}\text { Proteon } 1,2 \text { (MPM, Vectis, TVM, Skyline Ventures, Prism VentureWorks, } \\
\text { Intersouth, Bessemer, Devon Park, angel investors) }\end{array}$ & 50.0 & 2 & 28-May & & CuraGen & Celldex & 94.5 & 29-May \\
\hline & Cempra (Quaker BioVentures) & 46.0 & 3 & 14-May & & IDM Pharma & Takeda & 66.7 & 18-May \\
\hline & Aileron (SR One, Excel Medical) & 40.0 & 4 & 8-Jun & & & & & \\
\hline & Anaphore $^{3}$ (5AM Ventures, Versant, Apposite Capital) & 38.0 & 1 & 14-May & & & & & \\
\hline & Xanodyne (MPM) & 38.0 & NA & 4-Jun & & & & & \\
\hline \multirow[t]{8}{*}{$\begin{array}{l}\text { Licensing / } \\
\text { collaboration }\end{array}$} & Researcher & Investor & $\begin{array}{c}\text { Value } \\
\text { (\$ millions) }\end{array}$ & Deal descript & & & & & \\
\hline & Exelixis & Sanofi Aventis & $>1,161$ & \multicolumn{6}{|c|}{$\begin{array}{l}\text { Exclusive, worldwide rights to XL147 and XL765, oral phosphoinositide 3-kinase (PI3K) inhibitors in phase } \\
1 \mathrm{~b} / 2 \text { to treat cancer }\end{array}$} \\
\hline & Chroma & GlaxoSmithKline & $>1,000$ & \multicolumn{6}{|c|}{ Options to license exclusive, worldwide rights to four macrophage-targeted small-molecule programs } \\
\hline & Concert & GlaxoSmithKline & $>1,000$ & \multicolumn{6}{|c|}{$\begin{array}{l}\text { Options to license exclusive, worldwide rights to three deuterium-containing compounds, including protease inhibi- } \\
\text { tor CTP- } 518 \text {, which is expected to enter phase } 1 \text { for HIV in } 2 \mathrm{HO9}\end{array}$} \\
\hline & Cardiome & Merck & 700 & \multicolumn{6}{|c|}{ Rights to develop and commercialize intravenous and oral formulations of atrial fibrillation compound vernakalant } \\
\hline & Cytokinetics & Amgen & 650 & \multicolumn{6}{|c|}{$\begin{array}{l}\text { Exercises option to license exclusive, worldwide rights, except in Japan, to cardiac contractility program, which } \\
\text { includes cardiac myosin activator CK- } 1827452 \text { in phase } 2 \text { for heart failure }\end{array}$} \\
\hline & Globelmmune & Celgene & 540 & \multicolumn{6}{|c|}{$\begin{array}{l}\text { Option to license exclusive, worldwide rights to oncology candidates from Globelmmune, a developer of tar- } \\
\text { geted molecular immunogens dubbed Tarmogens }\end{array}$} \\
\hline & Catalyst & Wyeth & 500 & \multicolumn{6}{|c|}{$\begin{array}{l}\text { Exclusive, worldwide rights to develop and commercialize CB } 813 \text {, a recombinant human coagulation Factor } \\
\text { VIla that is in preclinical development for acute bleeding in hemophilia patients }\end{array}$} \\
\hline
\end{tabular}

